J. Clin. Chem. Clin. Biochem.

Vol. 17, 1979, pp. 613-618 \title{
Bestimmung der Hämoglobinkonzentration aus Messungen der Blut- und Plasmadichte mittels der
Biegeschwingermethode ${ }^{1}$ )
}

\author{
Von $H$. Hinghofer-Szalkay und $H$. Holzer
}

Aus dem Physiologischen Institut (Vorstand: Prof. Dr. Th. Kenner) und der Medizinischen Universitätsklinik (Vorstand: Prof. Dr. S. Sailer) der Universität Graz

(Eingegangen am 9. Februar/14. Juni 1979)

Zusammenfassung: Blutproben von Patienten wurden zur Messung der Blut- und Plasmadichte nach der Biegeschwingermethode, des Hämatokrit und der Hämoglobinkonzentration im Blut herangezogen. Es zeigte sich, daß aus den Dichtewerten allein die Hämoglobinkonzentration und der Hämatokrit mit ausreichender Genauigkeit berechnet werden können. Zusammen mit der Bestimmbarkeit der Gesamteiweißkonzentration aus der Dichte des Plasmas (Holzer et al (1978) J. Clin. Chem. Clin. Biochem. 16, 391-395) ergibt sich die Möglichkeit, mit zwei schnellen, einfachen und genauen Dichtemessungen drei klinisch relevante Parameter zu ermitteln, wobei insgesamt etwa $3 \mathrm{ml}$ Blut benötigt werden.

The calculation of hemoglobin concentration from blood and plasma densities, measured by the mechanical oscillator technique

Summary: Blood samples from patients were taken for the measurement of blood and plasma densities by the mechanical oscillator technique, and the hematocrit value and the hemoglobin content of the blood. The values for blood and plasma density permit the precise calculation of blood hemoglobin concentration and hematocrit. Thus, in addition to the calculation of total protein concentration of plasma (Holzer et al (1978) J. Clin. Chem. Clin. Biochem. 16, 391-395), measurement of the blood density yields two further relevant parameters. In total, only $3 \mathrm{ml}$ of blood are needed for the measurements.

\section{Einführung}

Im Rahmen von Untersuchungen über die Blutdichte mittels eines neuen Meßverfahrens (1-3) ergab sich die Frage nach der im Prinzip seit langem bekannten Beziehung zwischen Hämoglobingehalt und Dichte der roten Blutzzellen (4).

Blut- und Plasmadichte lassen sich mittels des von uns benützten Biegeschwingerprinzips schnell, einfâch und äußerst genau messen. Über die Ëignung der Methode zur Gesamteiweißbestimmung aus der Plasmadichte wurde schon früher berichtet (5). Wir überprüften nun, wie verlä̈ßlich bei zusätzlicher Messung der Blutdichte auch der Hämoğlobingehalt errechenbar ist und weiters, wie gut aus den Werten der Hämatokrit abschätzbar ist.

Dazu wurden von verschiedenen Probanden gewonnene Proben zu Messungen der Dichtewerte, der Hämogoglobin-

1) Unterstützt durch den Fọnds zưr Förderung der wissenschaftlichen Forschung. konzentration des Blutes und des Hämatokrit herangezogen. Die eingangs erwähnte Problemstellung wurde an Hand der so gewonnenen Daten untersucht.

\section{Methoden}

20 willkürlich ausgewählten männlichen Patienten einer medizinischen Klinik wurde nach Stauung am Oberarm jeweils $5 \mathrm{ml}$ venöses Blut aus einer Kubitalvene in sterile, zwei Tropfen Heparinlösung enthaltende Einmalspritzen entnommen und diese sofort auf einem Probenwender fixiert. Ein Teil des Blutes wurde zwecks Gewinnung von Plasma zentrifugiert und das Plasma ebenfalls in Einmalspritzen gefüllt.

Der Hämoglobingehalt wurde kolorimetrisch, jeweils doppelt oder dreifach, der Hämatokrit mittels Zentrifuge jeweils sechsfach bestimmt. Zur weiteren Berechnung wurden die Mittelwerte der Messungen verwendet.

Plasma- und Blutdichtewerte wurden mit einem Dichtemeßgerät DMA 55 der Firma Paar KG, Graz, untersucht. Die Messungen erfolgten bei $20,0^{\circ} \mathrm{C}$. Zur Thermostatisierung diente ein Ultrathermostat Type CB 60 der Firma Heto.

Die Blutproben wurden bis unmittelbar vor Füllung der Meßröhrchen für die Hämoglobin- und Hämatokritbestimmung sowie 
der Dichtemeßanordnung durch einen Probenwender in Bewegung gehalten, um Aggregation der Blutzellen und Senkungseffekte zu vermeiden.

Das Dichtemeßgerät arbeitet nach dem von Kratky, Leopold und Stabinger beschriebenen Prinzip (1). Die Probe wird in ein aus Glas bestehendes U-Rohr eingebracht, welches zu Schwingungen in seiner Eigenfrequenz angeregt wird. Je höher die Dichte und somit die Masse der Probe, desto langsamer oszilliert der gesamte Körper und umso länger währt ein Schwingungszyklus. Die Dauer dieser Periode wird vom Gerät gemessen und der Wert in die entsprechende Dichte der Probe umgerechnet. Eine detaillierte Beschreibung des Meßprinzips findet sich in 1.c. $(1,2)$.

In keinem Fall konnte während der Blutdichtemessung eine Senkung im Schwinger oder eine den Meßwert verfälschende Anreicherung von Blutzellen in der Schwingerspitze (6) beobachtet werden, die angezeigten Beträge waren während der für Temperaturangleich und Messung erforderlichen Zeit (weniger als eine Minute) stabil.

Die gemessenen Plasma- und Blutdichtewerte waren bei wiederholter Füllung des Meßschwingers jeweils aus derselben Spritze, nach Verwerfen der vorhergehend gemessenen Fraktion, auf $10^{-2} \mathrm{~g} / 1$ reproduzierbar. Auf mehrfache Bestimmungen der Dichtewerte konnte im Einzelfall daher verzichtet werden.

\section{Liste der verwendeten Symbole}

In den folgenden Texten und Darstellungen werden die nachstehenden Symbole verwendet:

$\rho \mathrm{p}$ : Plasmadichte (in $\mathrm{g} / \mathrm{l}$ )

$\rho_{\mathrm{B}}$ : Blutdichte (in $\mathrm{g} / \mathrm{l}$ ),

$\rho_{\mathrm{E}}$ : Erythrocytendichte, berechnet aus $\rho_{\mathrm{P}}, \rho_{\mathrm{B}}$ und Hämatokrit (in $\mathrm{g} / \mathrm{l}$ ),

$\rho_{\text {Eo }}$ : Dichte der ,hämoglobinleeren" Erythrocyten als Nullpunktdurchgang der Regressionsgeraden entsprechend Gleichung (Gl. 3) und Abbildung 1. Aus den Meßwerten ergab sich für $\rho_{\text {Eo }} 1008,52 \mathrm{~g} / \mathrm{l}$.

$\mathrm{c}_{\mathrm{Hb}}$ : Hämoglobinkonzentration im Blut, kolorimetrisch bestimmt (in $\mathrm{g} / \mathrm{l}$ );

$c_{\mathrm{Hb}}$ : Hämoglobinkonzentration in den Erythrocyten (in $\mathrm{g} / \mathrm{l}$ Erythrocyten), berechnet aus $\mathrm{c}_{\mathrm{Hb}_{\mathrm{B}}}$ und Hämatokrit nach Gleichung (Gl. 2),

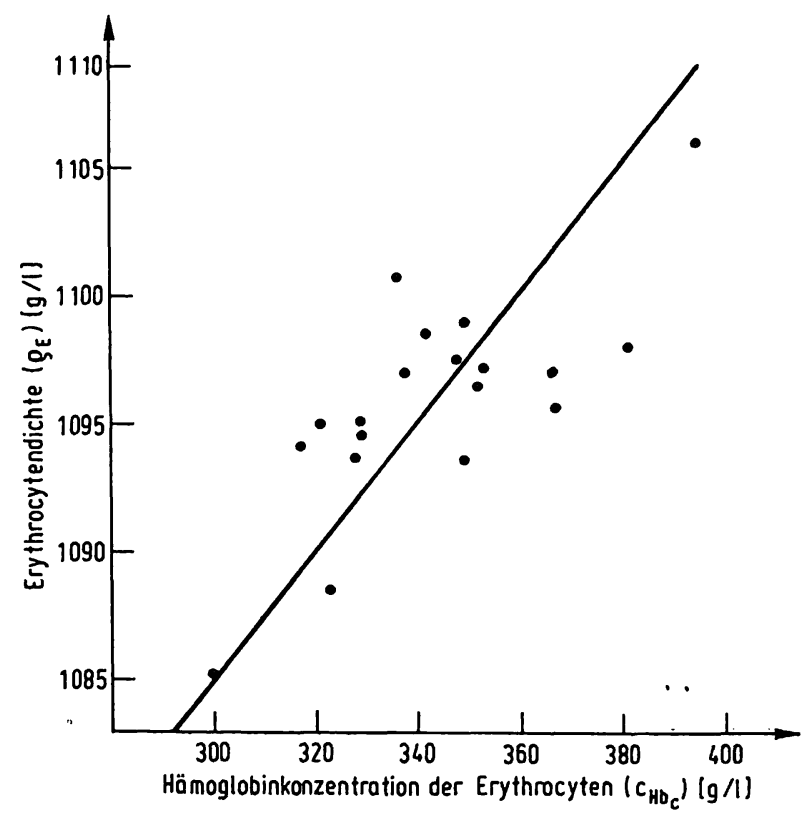

Abb. 1. Beziehung zwischen Erythrocytendichte $\left(\sigma_{\mathrm{E}}\right)$ und erythrocytärer Hämoglobinkonzentration ( $\mathrm{C}_{\mathrm{Hb}}$, beides in $\mathbf{g} / \mathbf{l}) . \mathbf{N}=20, \mathbf{r}=0,751$, Steigung der Regressionsgeraden $\mathrm{a}=0,25479$, Dichte bei $c_{\mathrm{Hb}_{\mathrm{C}}}=0\left(\sigma_{\mathrm{EO}}\right)=1008,52 \mathrm{~g} / \mathrm{l}$.
cHb : Hämoglobinkonzentration im Blut, berechnet nach Gleichung (Gl. 6) aus Blut- und Plasmadichte sowie Hämatokrit (in $\mathrm{g} / \mathrm{l}$ );

$\mathrm{c}_{\mathrm{H}} \mathrm{b}_{\mathrm{B}}$ : Hämoglobinkonzentration im Blut, berechnet nach Gleichung (GI. 8) aus Blut- und Plasmadichte (in $\mathrm{g} / \mathrm{I}$ );

Ht: Hämatokrit, ermittelt mit Hilfe der Hämatokritzentrifuge, in Volumenfraktion;

$\mathrm{Ht}_{\text {corr }}$ : „wahrer" Hämatokrit, berechniet nach l.c. (7) als Ht$0,04 \mathrm{Ht}$, in Volumenfraktion;

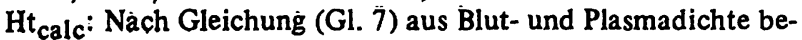
rechneter wahrer Hämatokrit, unter Zugrundelegung einer Erythrocytendichte von 1095,72 g/l (siehe Diskussion), in Volumenfraktion.

\section{Theorie und Auswertung}

Den Ansatz für die Berechenbarkeit der Hämoglobinkonżentration aus den Dichtewerten stellt die Überlegung dar, daß die Blutdichte dem gewichteten Mittel aus Plasmadichte und Blutzelldichte, vereinfacht Erythrocytendichte, entspricht (6):

$$
\rho_{\mathrm{B}}=\rho_{\mathrm{P}}\left(1-\mathrm{Ht}_{\mathrm{corr}}\right)+\rho_{\mathrm{E}} \cdot \mathrm{Ht}_{\text {corr }}
$$

Weiters läßt sich die Hämoglobinkonžentration im ,wahren “ Ery throcytenvolumen (erythrocy täre Hämoglobinkonzentration $\mathrm{c}_{\mathrm{Hb}_{\mathrm{C}}}$ ) aus der Beziehung

$$
\mathrm{c}_{\mathrm{Hb}_{\mathrm{C}}}=\frac{\mathrm{c}_{\mathrm{Hb}_{\mathrm{B}}}}{\mathrm{Ht}_{\text {corr }}}(\mathrm{g} / \mathrm{l})
$$

errechnen.

Die Annahme einer linearen Beziehung zwischen Erythrocytendichte und Hämoglobinkonzentration der Erythrocyten (4) ließ sich bestätigen (siehe Ergebnisse) und kann in Form folgender Gleichung ausgedrückt werden:

$$
\rho_{\mathrm{E}}=\rho_{\mathrm{EO}_{\mathrm{O}}}+\mathrm{a} \cdot \mathrm{c}_{\mathrm{Hb}} \mathrm{C}
$$

Dabei bedeuten a die Steilheit der Regressionsgeraden und $\rho_{\mathrm{E}}$ die Dichte bei $\mathrm{c}_{\mathrm{Hb}}=0$.

Aus den Gleichungen (Gl. 2) und (Gl. 3) ergibt sich für die Hämoglobinkonzentration des Blutes die Beżiẹung

$$
\mathrm{c}_{\mathrm{Hb}_{\mathrm{B}}}=\frac{1}{\mathrm{a}}\left(\rho_{\mathrm{E}}-\rho_{\mathrm{Eo}}\right) \mathrm{Ht} \text { corr }
$$

Aus Gleichung (Gl. 1) berechnet sich die Erythrocytendichte als

$$
\rho_{\mathrm{E}}=\frac{\rho_{\mathrm{B}}-\rho_{\mathrm{P}}\left(1-\mathrm{Ht}_{\mathrm{corr}}\right)}{\mathrm{Ht}_{\text {corr }}},
$$

und nach Einsetzen dieses Ausdrucks in Gleichung (Gl. 4) ergibt sich:

$$
\mathrm{c}_{\mathrm{Hb}_{\mathrm{B}}}=\frac{1}{\mathrm{a}}\left[\rho_{\mathrm{B}}-\rho_{\mathrm{P}}\left(1-\mathrm{Ht}_{\mathrm{corr}}\right)\right]-\frac{1}{\mathrm{a}}\left(\rho_{\mathrm{Eo}} \cdot \mathrm{Ht} \text { corr }\right)
$$

\section{Ergebnisse}

In Tabelle 1 findet sich eine Zusammenstellung der gemessenen Daten. Die Dichte der Plasmen $\left(\rho_{\mathrm{P}}\right)$ betrug durchschnittlich 1025,60 g/l (höchster Wert 1030,17 g/l, Probe Nr. 13, niedrigster Wert $1021,19 \mathrm{~g} / \mathrm{l}$, Probe Nr. 4). Diese Werte sind überwiegend von der Gesamteiweißkon- 
Tab. 1. Gemessene Daten. Zur Erklärung der Abkürzungen und Dimensionen siehe den betreffenden Abschnitt.

\begin{tabular}{|c|c|c|c|c|}
\hline $\begin{array}{l}\text { Probe } \\
\text { Nr. }\end{array}$ & $\rho \mathbf{P}$ & $\rho_{\mathrm{B}}$ & $\mathrm{Ht}$ & ${ }^{c_{H} b_{B}}$ \\
\hline $\begin{array}{r}1 \\
2 \\
3 \\
4 \\
5 \\
6 \\
7 \\
8 \\
9 \\
10 \\
11 \\
12 \\
13 \\
14 \\
15 \\
16 \\
17 \\
18 \\
19 \\
20\end{array}$ & $\begin{array}{l}1024,97 \\
1025,01 \\
1023,82 \\
1021,19 \\
1023,07 \\
1024,93 \\
1024,59 \\
1025,52 \\
1027,16 \\
1026,66 \\
1025,86 \\
1027,11 \\
1030,17 \\
1025,00 \\
1025,46 \\
1029,27 \\
1022,20 \\
1024,65 \\
1029,52 \\
1025,91\end{array}$ & $\begin{array}{l}1052,42 \\
1053,25 \\
1051,68 \\
1041,00 \\
1055,13 \\
1053,92 \\
1047,88 \\
1055,00 \\
1047,41 \\
1057,08 \\
1057,10 \\
1049,00 \\
1057,60 \\
1053,51 \\
1051,43 \\
1051,10 \\
1055,90 \\
1049,04 \\
1050,92 \\
1052,51\end{array}$ & $\begin{array}{l}0,3856 \\
0,3997 \\
0,3988 \\
0,2592 \\
0,4478 \\
0,3728 \\
0,3673 \\
0,4470 \\
0,3010 \\
0,4503 \\
0,4703 \\
0,3712 \\
0,4210 \\
0,4122 \\
0,3970 \\
0,3450 \\
0,4850 \\
0,3680 \\
0,4000 \\
0,3970 \\
\end{array}$ & $\begin{array}{l}129,5 \\
131 \\
134,5 \\
83,5 \\
149,5 \\
141 \\
114,5 \\
136 \\
102 \\
146 \\
145 \\
115 \\
154 \\
145 \\
133 \\
109 \\
153 \\
116 \\
115 \\
140 \\
\end{array}$ \\
\hline $\begin{array}{l}\bar{x} \\
s_{x}\end{array}$ & $\begin{array}{r}1025,60 \\
2,92\end{array}$ & $\begin{array}{r}1052,14 \\
4,00\end{array}$ & $\begin{array}{l}0,3949 \\
0,0542\end{array}$ & $\begin{array}{r}129,6 \\
18,8\end{array}$ \\
\hline
\end{tabular}

zentration bestimmt (5), welche demnach zwischen etwa 57 und $89 \mathrm{~g} / 1$ (Mittelwert $73 \mathrm{~g} / \mathrm{l}$ ) lag. Die Dichte der Vollblute $\left(\rho_{B}\right)$ betrug im Mittel $1052,14 \mathrm{~g} / 1$ mit Extremen von 1041,00 (Probe Nr. 4) und 1057,60 g/l (Probe Nr. 13).

Die Hämatokritwerte $(\mathrm{Ht})$ wurden bei jeder Probe sechsfach bestimmt; die Mittelwerte sind in Tabelle 1 angegeben und dienten zur weiteren Berechnung. Die Stan- dardabweichungen aus den je sechs Meßresultaten betrugen zwischen 0,0005 und 0.0045 Volumenfraktionen ( 0.05 bis 0.45 Hämatokrit-\%).

Die kolorimetrisch ermittelte Hämoglobinkonzentration im Blut $\left(\mathrm{c}_{\mathrm{Hb}_{\mathrm{B}}}\right)$ betrug im Durchschnitt $129,6 \mathrm{~g} / 1$, der höchste Wert war 154 (Probe Nr. 13), der geringste 83,5 g/l (Probe Nr. 4).

Tabelle 2 zeigt eine Ubersicht über die berechneten Werte. Zunächst wurden die gemessenen Hämatokritwerte $(\mathrm{Ht})$ für $4 \%$ "trapped plasma" (7) korrigiert. Die so ermittelten Werte für den ,wahren" Hämatokrit $\left(\mathrm{Ht}_{\text {corr }}\right)$ bewegten sich zwischen 0,2488 (Probe Nr. 4) und 0,4656 (Probe Nr. 17), das Mittel lag bei 0,3790 (Angaben in Volumenfraktion).

Die (durchschnittliche) Erythrocytendichte $\left(\rho_{E}\right)$ jeder einzelnen Probe konnte nicht direkt gemessen werden, da auch nach intensiver Zentrifugation Reste von Plasma zwischen den Zellen verbleiben. Sie wurde durch lineare Extrapolation auf den Hämatokritwert 1 bestimmt, indem der Plasmadichte jeder Probe der Hämatokritwert 0 und ihrer Blutdichte der ,wahre " Hämatokritwert $\mathrm{Ht}_{\text {corr }}$ zugeordnet wurden.

Die so ermittelten Beträge von $\rho_{\mathrm{E}}$ schwankten zwischen 1085,25 (Probe Nr. 19) und 1105,93 g/l (Probe Nr. 6), im Schnitt lagen sie bei $1095,72 \mathrm{~g} / \mathrm{l}$.

Die erythrocytäre Hämoglobinkonzentration $\left(\mathrm{c}_{\mathrm{HB}_{\mathrm{c}}}\right)$ wurde nach Gleichung (Gl. 2) ermittelt. Der gefundene Minimalbetrag war 299,5 Gramm Hämoglobin pro Liter Erythrocyten (Probe Nr. 19), der höchste 394,0 g/l (Probe Nr. 6), als Mittelwert wurden 342,3 g/l gefunden,

Tab. 2. Bereçhnete Daten. Zur Erklärung der Abkürzungen und Dimensionen siehe den betreffenden Abschnitt.

\begin{tabular}{|c|c|c|c|c|c|c|}
\hline Probe & $\mathbf{H t}_{\text {corr }}$ & $\rho_{\mathbf{E}}$ & $\mathrm{c}_{\mathrm{Hb}} \mathrm{C}$ & $\mathrm{c}_{\mathrm{H}}^{+} \mathrm{b}_{\mathrm{B}}$ & $\mathrm{Ht}_{\text {calc }}$ & $c_{\mathrm{Hb}_{B}}^{++}$ \\
\hline $\begin{array}{r}1 \\
2 \\
3 \\
4 \\
5 \\
6 \\
7 \\
8 \\
9 \\
10 \\
11 \\
12 \\
13 \\
14 \\
15 \\
16 \\
17 \\
18 \\
19 \\
20\end{array}$ & $\begin{array}{l}0,3710 \\
0,3837 \\
0,3828 \\
0,2488 \\
0,4299 \\
0,3579 \\
0,3526 \\
0,4291 \\
0,2890 \\
0,4323 \\
0,4515 \\
0,3562 \\
0,4042 \\
0,3955 \\
0,3811 \\
0,3312 \\
0,4656 \\
0,3533 \\
0,3840 \\
0,3811\end{array}$ & $\begin{array}{l}1098,96 \\
1098,61 \\
1096,60 \\
1100,81 \\
1097,65 \\
1105,93 \\
1090,64 \\
1094,22 \\
1097,23 \\
1097,03 \\
1095,05 \\
1088,56 \\
1098,03 \\
1097,09 \\
1093,60 \\
1095,18 \\
1094,58 \\
1093,68 \\
1085,25 \\
1095,71\end{array}$ & $\begin{array}{l}349,1 \\
341,4 \\
351,4 \\
335,6 \\
347,8 \\
394,0 \\
324,7 \\
316,9 \\
352,9 \\
337,7 \\
321,2 \\
322,9 \\
381,0 \\
366,6 \\
349,0 \\
329,1 \\
328,6 \\
328,3 \\
299,5 \\
367,4\end{array}$ & $\begin{array}{r}131,7 \\
135,7 \\
132,3 \\
90,1 \\
150,4 \\
136,8 \\
113,6 \\
144,3 \\
100,6 \\
150,2 \\
153,3 \\
111,9 \\
142,0 \\
137,5 \\
127,3 \\
112,7 \\
157,3 \\
118,1 \\
115,6 \\
130,4\end{array}$ & $\begin{array}{l}0,3880 \\
0,3994 \\
0,3875 \\
0,2658 \\
0,4413 \\
0,4095 \\
0,3274 \\
0,4199 \\
0,2954 \\
0,4405 \\
0,4472 \\
0,3190 \\
0,4185 \\
0,4031 \\
0,3696 \\
0,3285 \\
0,4584 \\
0,3432 \\
0,3233 \\
0,3810\end{array}$ & $\begin{array}{r}132,8 \\
136,7 \\
132,6 \\
91,0 \\
151,0 \\
140,2 \\
112,1 \\
143,7 \\
101,1 \\
150,8 \\
153,0 \\
109,2 \\
143,2 \\
138,0 \\
126,5 \\
112,4 \\
156,9 \\
117,5 \\
110,6 \\
130,4\end{array}$ \\
\hline $\begin{array}{l}\overline{\mathbf{x}} \\
\mathbf{s}_{\mathbf{x}}\end{array}$ & $\begin{array}{l}0,3790 \\
0,0520\end{array}$ & $\begin{array}{r}1095,72 \\
4,38\end{array}$ & $\begin{array}{r}342,3 \\
22,9\end{array}$ & $\begin{array}{r}129,6 \\
18,2\end{array}$ & $\begin{array}{l}0,3783 \\
0,0548\end{array}$ & $\begin{array}{r}129,5 \\
18,7\end{array}$ \\
\hline
\end{tabular}


was mit Literaturwerten übereinstimmt (8). Die Beziehung zwischen Erythrocytendichte und ery throcytärer Hämoglobinkonzeritration kann als linear angesehen werden und ist, entsprechend den in Tabelle 2 angegebenen Daten, in Abbildung 1 dargestellt. Die Regression kann in Form von Gleichung (Gl. 3) ausgedrückt werden. Der Korrelationskoeffizient betrug 0.751; für a ergab sich der Wert 0.25479 und fur $\rho_{\text {Eo }} 1008.52 \mathrm{~g} / 1$, was etwas über der Dichte einer eiweißlosen Körperflüssigkeit $\left(1005-1006 \mathrm{~g} / 1\right.$ bei $\left.20^{\circ} \mathrm{C}\right)$ liegt und daher für die Dichte „leerer“ Erythrocyten als plausibel erscheint.

Auf Grund der gefundenen Zahlenwerte für a und $\rho_{\text {Eo }}$ kann Gleichung (Gl. 5) in Form folgender Formel angeschrieben werden:

$$
\begin{aligned}
& \mathrm{c}_{\mathrm{Hb}}^{+} \mathrm{B}_{\mathrm{B}}=3,9248\left[\rho_{\mathrm{B}}-\rho_{\mathrm{P}}\left(1-\mathrm{Ht}_{\text {corr }}\right)\right] . \\
& -3958,24 \mathrm{Ht}_{\text {corr }}
\end{aligned}
$$

Durch Verwendung dieser Gleichung ergeben sich die unter $\mathrm{c}_{\mathrm{H} b_{\mathrm{B}}}^{+}$aufscheinenden Werte der Tabelle 2. Der Durchschnitt dieser aus ,wahrem “ Hämatokrit, Blutund Plasmadichte errechneten Werte liegt bei $129,6 \mathrm{~g} / \mathrm{l}$, ist also identisch mit dem der gemessenen $\mathrm{c}_{\mathrm{Hb}_{\mathrm{B}}}$-Werte. Die mittlere Abweichung der gerechneten von den gemessenen Beträgen betrug 4,6 $\mathrm{g} / 1$, das sind 3,5\% des durchschnittlichen Meßwertes. Die größte Abweichung betrug 12,0 g/l (Probe Nr. 13), das sind 9,3\% des durchschnittlichen Meßwertes.

In Abbildung 2 sind die nach Formel (Gl. 6) berechneten Werte $\mathrm{c}_{\mathrm{Hb}}^{+}$in, Abhängigkeit von den kolorimetrisch bestimmten $\left(\mathrm{C}_{\mathrm{Hb}_{\mathrm{B}}}\right)$ aufgetragen. Der Korrelationskoeffizient beträgt $0,953(\mathrm{~N}=20)$.

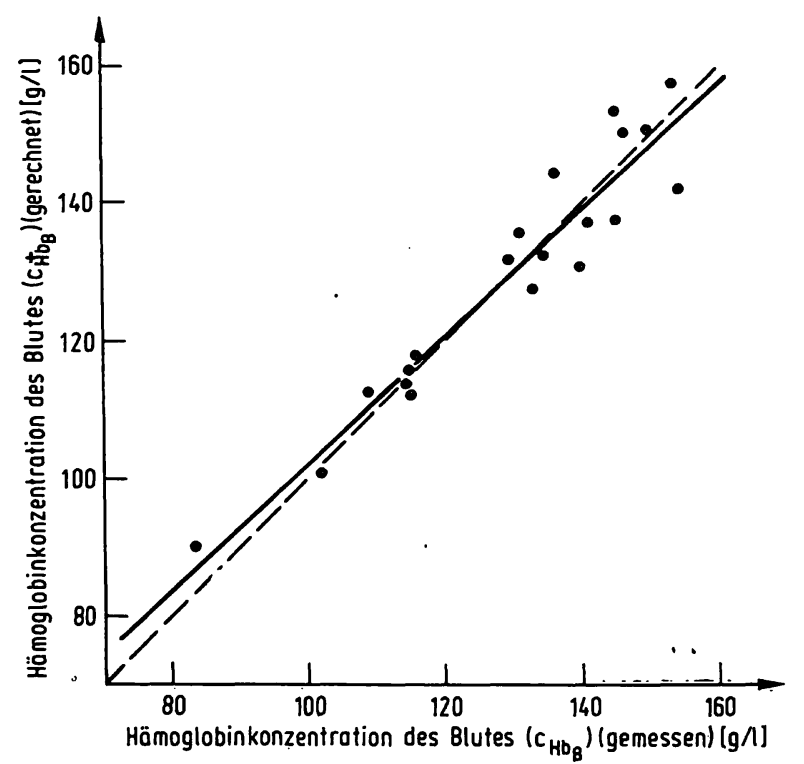

Abb. 2. Beziehung zwischen gemessenen $\left(c_{H b B}\right)$ und nach Formel (GL. 6) gerechneten Hämoglobinwerten ( $\mathrm{C}_{\mathrm{Hb}}^{+}$); jeweils in $\mathrm{g} / \mathrm{l}$. Strichliert: Identitätsgerade; ausgezogen: Regressionsgerade.

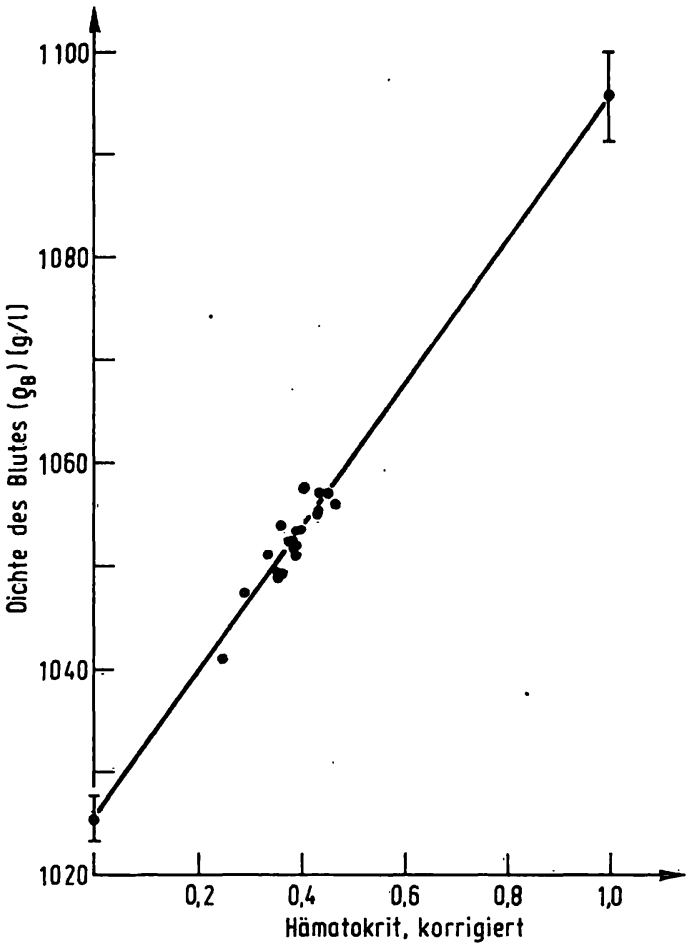

Abb. 3. Beziehung zwischen Blutdichte und Hämatokrit. Für die Berechnung wurden nur die Blutdichte-HämatokritWertepaare verwendet; nicht aber die ebenfalls gezeigten Plasma- und Erythrocytendichtewerte, welche den Durchschnitt der jeweils 20 Einzelbeträge angeben $\mathrm{N}=20, \mathrm{r}=0,898$. Die Steigung der Regressionsgeraden $(69,1 \mathrm{~g} / 1$ : Volumenfraktion) entspricht der mittleren Differenz zwischen Blut- und Plasmadichten.

Die Beziehung zwischen Dichte des Blutes und "wahrem" Hämatokrit, die sich aus den Werten unserer 20 Proben ergab, ist in Abbildung 3 dargestellt. Der Korrelationskoeffizient beträgt $0,898(\mathrm{~N}=20)$. Im Bild sind auch die Mittelwerte und Standardabweichungen für Plasmaund Erythrocytendichten eingetragen. Die Regressionsgerade läuft bei $\mathrm{Ht}_{\text {corr }}=0$ durch den Wert 1025,96 und bei $\mathrm{Ht}_{\text {corr }}=1$ durch 1095,04, was den Mittelwerten für $\rho_{\mathrm{P}}$ und $\rho_{\mathrm{E}}$ ziemlich genau entspricht, obwohl letztere nicht in die Berechnung der Regresșion aufgenommen wurden.

Berechnet man für jede einzelne Probe den Häämatokritwert aus der Blutdichte unter Zugrundelegung der individuellen Plasmadichte für $\mathrm{Ht}=0$ und einer mittleren Erythrocytendichte von $1095,72 \mathrm{~g} / 1$ für $\mathrm{Ht}_{\text {corr }}=1$ (siehe Diskusssion) nach der Gleichung

$$
\mathrm{Ht}_{\text {corr }}=\frac{\rho_{\mathrm{B}}-\rho_{\mathrm{P}}}{\rho_{\mathrm{E}}-\rho_{\mathrm{P}}},
$$

so ergeben sich die als $\mathrm{Ht}_{\text {calc }}$ bezeichneten Werte in Tabelle 2. Ihr Durchschnitt beträgt 0,3783, was um 0,007 unter dem der Werte $\mathrm{Ht}_{\text {corr }}$ liegt. Die mittlere $\mathrm{Ab}$ weichung der aus Dichtewerten berechneten von den "wahren" Hämatokritwerten betrug 0,0161 (entspricht $4,2 \%$ des durchsschnittlichen Meßwerts), die höchste 0,0607 , entsprechend $16,0 \%$ des mittleren Meßwerts (Probe Nr. 19). 


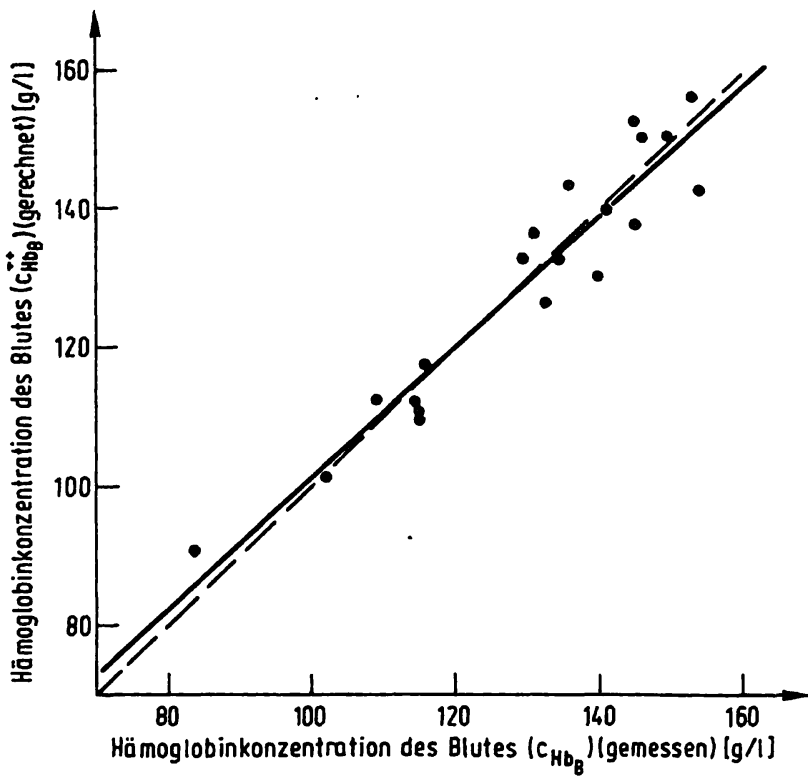

Abb. 4. Beziehung zwischen gemessenen $\left(c_{\mathrm{Hb}_{B}}\right)$ und nach Formel (Gl. 8) gerechneten Hämoglobinwerten ( $\mathrm{CHb}_{\mathrm{B}}^{++}$); jeweils in $\mathrm{g} / \mathrm{L}$. Strichliert: Identitätsgerade; ausgezogen: Regressionsgerade.

Um zu prüfen, wie gut sich die kalkulierten Hämatokritwerte für die Berechnung des Hämoglobinwertes nach Gleichung (Gl. 5) eignen, wurde die Relation zwischen berechneten und gemessenen Hämoglobinwerten ermittelt, die sich ergibt, wenn man in Gleichung (Gl. 6) nicht die gemessenen, sondern die aus den Dichtewerten gerechneten Hämatokritbeträge $\left(\mathrm{Ht}_{\text {calc }}\right)$ einsetzt. Diese Berechnung erfolgt durch Verwendung einer Formel, die sich nach Einsetzen von (Gl. 7) in (Gl. 6) ergibt und lautet:

$$
\begin{aligned}
\mathrm{c}_{\mathbf{H b}_{\mathrm{B}}}^{++}= & 3,9248\left[\rho_{\mathrm{B}}-\rho_{\mathrm{P}}\left(1-\frac{\rho_{\mathrm{B}}-\rho_{\mathrm{P}}}{1095,72-\rho_{\mathrm{P}}}\right)\right]- \\
& \frac{3958,24\left(\rho_{\mathrm{B}}-\rho_{\mathrm{P}}\right)}{1095,72-\rho_{\mathrm{P}}}
\end{aligned}
$$

So ergeben sich die neuen, in Tabelle 2 unter $\mathrm{C}_{\mathrm{Hb}_{\mathrm{B}}}^{++} \mathrm{ge}$ zeigten Hämoglobinwerte für das Blut. Deren Mittelwert beträgt $129,5 \mathrm{~g} / 1$, liegt also um $0,1 \mathrm{~g} / 1$ unter dem der gemessenen Werte. Die mittlere Abweichung der solchermaßen berechneten von den kolorimetrisch bestimmten Werten betrug $4,4 \mathrm{~g} / 1$, das sind $3,4 \%$ des durchschnittlichen Meß̂wertes, die maximale 10,8 g/1 (Probe Nr. 13), das sind $8,3 \%$ des mittleren Meßwertes.

Abbildung 4 żeigt diese Beziehung zwischen kolorimetrisch ermittelten Hämoglobinwerten ( $\mathrm{c}_{\mathrm{Hb}_{\mathrm{B}}}$, Abszisse) und den aus Blut- und Plasmadịchte nach Formel (Gl. 8) errechneten $\left(\mathrm{C}_{\mathrm{Hb}}^{+}\right.$, Ordinate). Der Korrelationskoeffizient beträgt $0,952(\mathrm{~N}=20)$.

\section{Diskusșion}

Die hier vorgelegten Ergebnisse belegen eine für klinische Zwecke aușeichend genaue Berechenbarkeit von Hämoglobin- und Hämatokritwerten aus der Dichtemessung von
Blut und dazugehörigem Plasma mittels der Biegeschwingermethode.

Nachdem schon früher gezeigt wurde, daß sie zur Ermittlung der Gesamteiweißkonzentration aus der Plasmadichte sehr gut geeignet ist (5), ergibt sich die Möglichkeit, zwei weitere klinisch relevante Kenngrößen, nämlich Hämoglobinkonzentration und Hämatokrit, aus der zusätzlichen Messung der Blutdichte zu bestimmen.

Die Hämatokritmessung mittels Zentrifuge stellt im Vergleich zur Präzision der Dichtemessungen den größten Unsicherheitsfaktor dar. Die Ablesung des Einzelwertes ist mit einem Fehler behaftet, der bis zu $1 \%$ des Meßwertes betragen kann.

Eine wesentlich genauere Ermittlung des Hämatokrit wäre nach Formel (Gl. 7) möglich, was allerdings eine bekannte Erythrocy tendichte voraussetzt. Diese kann aber, wie schon diskutiert, nicht direkt gemessen und zur Berechnung von $\mathrm{Ht}_{\text {corr }}$ daher nicht als bekannt vorausgesetzt werden. Aus diesem Grund nahmen wir bei allen Proben für $\rho_{E}$ den Mittelwert aller zunächst berechneten Werte $(1095,72 \mathrm{~g} / \mathrm{l})$ an. Auf diese Weise ergaben sich die berechneten Hämatokritwerte. Es zeigte sich, daß bie diesem Vorgehen etwa die gleiche Fehlerbreite bei der Ermittlung der Hämoglobinkonzentration im Blut resultiert wie bei Verwendung der gemessenen Hämatokritwerte. Das heißt, daß sich in diesem Zusammenhang bei Messung der Blut- und Plasmadichte die Hämatokritmessung erübrigt.

Da die Bestimmung der Blut- und Plasmadichte nach der Biegeschwingermethode gleichzeitig die Vorteile hoher Genauigkeit (besser als $10^{-2} \mathrm{~g} / \mathrm{l}$ ), geringem Zeitaufwand (insgesamt etwa 2 Minuten) und geringem benötigten Probenvolumen (jeweils unter $1 \mathrm{ml}$ ) bietet und aus den Werten der Hämoglobingehalt des Blutes, sein Hämatokrit und schließlich die Gesamteiweißkonzentration im Plasma (5) mit hinlänglicher Genauigkeit berechenbar sind, scheint uns die Methode für den Einsatz im klinischen Routinelabor geeignet zu sein.

Bezüglich möglicher Fehlerquellen sei schließlich der Einfluß der Leukocytenzahl auf die Bestimmung der Blut-Hämoglobinkonzentration aus Blut- und Plasmadichte diskutiert. Es sei vorweggenommen, daß dieser Faktor üblicherweise keinen großen Beitrag zu Abweichungen der gerechneten von den gemessenen Werten liefert. Dies kann am besten an Hand eines konkreten Rechenbeispiels gezeigt werden: In einem hämatologischen Extremfall (Leukämie mit schwerer Anämie kombiniert) spielt die Dichte der Leukocyten, welche nach unseren Messungen mit etwa $1055 \mathrm{~g} / \mathrm{l}$ angenommen werden kann, gewichtet mit dem Leukocyten-Hämatokrit, eine wesentliche Rolle als Komponente der Gesamtblutdichte. Nimmt man beispielsweise einen volumsmäßigen Anteil der Leukocyten von 0,05 Volumenfraktion bei einem Erythrocy ten-Hämatokrit von 0,15 Volumenfraktion an und legt eine Plasmadichte von 
$1025 \mathrm{~g} / \mathrm{l}$ und eine Erythrocytendichte von $1095 \mathrm{~g} / \mathrm{l} \mathrm{zu}-$ grunde, so errechnet sich entsprechend Gleichung (Gl. 8) ein Blut-Hämoglobinwert von $59,3 \mathrm{~g} / \mathrm{l}$ bei einem tatsächlichen Wert von $51,0 \mathrm{~g} / 1$, einen $\mathrm{c}_{\mathrm{Hb}_{\mathrm{C}}}$-Wert von $340 \mathrm{~g} / \mathrm{l}$ vorausgesetzt. Bei einem noch höheren Leukocytenanteil, z.B. 0,15 Volumenfraktion, erhöht sich $\mathrm{c}_{\mathrm{Hb}_{\mathrm{B}}}^{++}$in diesem Beispiel immerhin auf 72,2 g/l. Das entspräche einem Fehler von 21,2 g/1 Hämoglobinkonzentration; die schwere Anämie bzw. der niedrige Hämoglobinwert würden allerdings dennoch angezeigt werden.

\section{Literatur}

1. Kratky, O., Leopold, H. \& Stabinger, H.: (1969), Z. Angew. Physik 27, 273-277.

2. Kenner, T., Leopold, H. \& Hinghofer-Szalkay, Ḧ.: (1977), Pflügers Arch. 370, 25-29.

3. Kenner, T., Hinghofer-Szalkay, H. \& Leopold, H.: (1978), Cardiovasc. Pulm. Dyn. 71,283-290.

4. van Slyke, D. D., Phillips, R. A., Dole, V. P., Hamilton, P. B., Archibald, R. M. \& Plazin, J.: (1950), J. Biol. Chem. 183, 349-360.
Aus diesen Abschätzungen geht hervor, daß auch bei stark abweichenden hämatologischen Zustandsvariablen aus $\rho_{\mathrm{P}}$ und $\rho_{\mathrm{B}}$ eine verhältnismäßig genaue Hämoglobinwertberechnung möglich ist.

Tatsächlich konnten wir Abweichungen żwischen $\mathrm{c}_{\mathrm{Hb}_{\mathrm{B}}}$ und $\mathrm{c}_{\mathrm{Hb}_{\mathrm{B}}}^{++}$(z. B. Probe 13) nicht mit auffälligen Veränderungen im Blutbild korrelieren. Andererseits gibt bei genauer Bestimmung des Hämatokrit der ermittelte Erythrocytendichtewert über $\mathrm{c}_{\mathrm{Hb}_{\mathrm{C}}}$ genau Aufschluß.

5. Holzer, H., Leopold, H., Hinghofer-Szalkay, H., StübchenKirchner, H. \& Maurer, E.: (1978), J. Clin. Chem. Clin. Biochem. 16;391-395.

6. Leopold, H., Hinghofer-Szalkay, H., Kenner, T. \& Holzer, H.: (1978), Biomed. Technik 23,99-103.

7. Dill, D. B. \& Costill, D. L.: (1974), J. Appl. Physiol. 37,247248.

8. Wintrobe, M. M. (Ed.): Clinical Hematology, 6. Ed. Philadelphia: Lea \& Febiger 1968.

Dr. H. Hinghofer-Szalkay

Physiologisches Institut der Universität A-8010 Graz, Harrachgasse 21 